\title{
Growth and fatty acid characterization of microalgae isolated from municipal waste-treatment systems and the potential role of algal-associated bacteria in feedstock production
}

Kevin Stemmler, Rebecca Massimi, Andrea E Kirkwood

Much research has focused on growing microalgae for biofuel feedstock, yet there remain concerns about the feasibility of freshwater feedstock systems. To reduce cost and improve environmental sustainability, an ideal microalgal feedstock system would be fed by municipal, agricultural or industrial wastewater as a main source of water and nutrients. Nonetheless, the microalgae must also be tolerant of fluctuating wastewater quality, while still producing adequate biomass and lipid yields. To address this problem, our study focused on isolating and characterizing microalgal strains from three municipal wastewater treatment systems for their potential use in biofuel feedstock production. Most of the 19 isolates from wastewater grew faster than two culture collection strains under mixotrophic conditions, particularly with glucose. The fastest growing wastewater strains included the genera Chlorella and Dictyochloris. The fastest growing microalgal strains were not necessarily the best lipid producers. Under photoautotrophic and mixotrophic growth conditions, single strains of Chlorella and Scenedesmus each produced the highest lipid yields, including those most relevant to biodiesel production. A comparison of axenic and non-axenic versions of wastewater strains showed a notable effect of commensal bacteria on fatty acid composition. Strains grown with bacteria tended to produce relatively equal proportions of saturated and unsaturated fatty acids, which is an ideal lipid blend for biodiesel production. These results not only show the potential for using microalgae isolated from wastewater for growth in wastewater-fed feedstock systems, but also the important role that commensal bacteria may have in impacting the fatty acid profiles of microalgal feedstock. 
Growth and fatty acid characterization of microalgae isolated from municipal wastetreatment systems and the potential role of algal-associated bacteria in feedstock production

\author{
Submitted by: \\ Kevin Stemmler, Rebecca Massimi, and Andrea E. Kirkwood ${ }^{1}$ \\ Faculty of Science \\ University of Ontario Institute of Technology \\ 2000 Simcoe Street North \\ Oshawa, Ontario
}

Canada

L1H 7K4

${ }^{1}$ Corresponding Author:

e-mail: andrea.kirkwood@uoit.ca

tel: 905.721 .8668

fax: 905.721 .3304 


\section{Abstract}

Much research has focused on growing microalgae for biofuel feedstock, yet there remain concerns about the feasibility of freshwater feedstock systems. To reduce cost and improve environmental sustainability, an ideal microalgal feedstock system would be fed by municipal, agricultural or industrial wastewater as a main source of water and nutrients. Nonetheless, the microalgae must also be tolerant of fluctuating wastewater quality, while still producing adequate biomass and lipid yields. To address this problem, our study focused on isolating and characterizing microalgal strains from three municipal wastewater treatment systems (2 activated sludge and 1 aerated-stabilization basin systems) for their potential use in biofuel feedstock production. Most of the 19 isolates from wastewater grew faster than two culture collection strains under mixotrophic conditions, particularly with glucose. The fastest growing wastewater strains included the genera Chlorella and Dictyochloris. The fastest growing microalgal strains were not necessarily the best lipid producers. Under photoautotrophic and mixotrophic growth conditions, single strains of Chlorella and Scenedesmus each produced the highest lipid yields, including those most relevant to biodiesel production. A comparison of axenic and non-axenic versions of wastewater strains showed a notable effect of commensal bacteria on fatty acid composition. Strains grown with bacteria tended to produce relatively equal proportions of saturated and unsaturated fatty acids, which is an ideal lipid blend for biodiesel production. These results not only show the potential for using microalgae isolated from wastewater for growth in wastewater-fed feedstock systems, but also the important role that commensal bacteria may have in impacting the fatty acid profiles of microalgal feedstock. 
22

23

24

25

\section{Introduction}

World energy consumption of petroleum is currently estimated to be 4.4 billion tonnes per year to fuel electricity, automobiles, and industrial processes (Roddy, 2013). To meet the growing global energy demand, nations worldwide have considered renewable sources of fuel to offset the dependence on non-renewable sources like fossil fuels. Algal biofuels are a promising source of renewable energy since production is non-seasonal, and yields are not limited to one or two harvests per year (da Silva et al., 2009). When compared to other biofuel feedstocks like corn or switchgrass, algal biofuel crops possess 6-12 times greater yearly energy production (Sandefur, Matlock \& Costello, 2011). An additional benefit of algal feedstock relates to the variety of possible conversion processes such as direct combustion, pyrolysis, or chemical conversion. Algal biomass can be converted to fuels like hydrogen, oil, or even raw electricity (Brennan \& Owende, 2010; Tsukahara \& Sawayama, 2005).

Research in algal biofuels has been primarily directed towards improving algal biomass yield or increasing lipid content. Researchers have determined optimal temperatures, light conditions, and nutrients to obtain high growth rates for many strains (Chinnasamy et al., 2010;

Price, Yin \& Harrison, 1998). These parameters are not the same for each strain of microalgae, and as a result, they need to be optimized individually. One challenging aspect of growing microalgae compared to terrestrial plant crops is the sizeable amount of water required to sustain yields. To grow algal biofuel-feedstock at large-scale cultivation would require approximately 1.5 million liters of water per hectare (Cho et al., 2007). If grown in open ponds, the evaporation loss would be 7-11 million liters of water per hectare per year (Cho et al., 2007). This requirement is juxtaposed against the prediction that $66 \%$ of the world's population will be residing in regions without access to drinking water by 2025 (Mehanna et al., 2010). 
45

46

47

Additionally, Sandstrom (1995) showed that a 15\% reduction in precipitation due to climate change may result in a 40-50\% reduction in aquifer water recharge. As such, a major stumbling block for the economic and environmental feasibility of algal-based biofuel production is the potentially unsustainable requirement for freshwater.

In recent years, research has started to focus on exploiting wastewater rather than freshwater as a growth medium for algal feedstock production. Wastewater, particularly municipal and agricultural, typically contain elevated levels of essential nutrients such as nitrogen and phosphorous. For example, nitrogen and phosphorus concentrations in municipal wastewaters can range from $10-100 \mathrm{mg} \cdot \mathrm{L}^{-1}$ and $>1000 \mathrm{mg} \cdot \mathrm{L}^{-1}$ in agricultural wastewater (de la Noue, Laliberte \& Proulx, 1992). Not only does wastewater serve as a good growth medium for microalgae, but conversely, algal growth in wastewater can serve as a tertiary treatment option (de la Noue et al., 1992; Lau et al. 1995; Woertz et al. 2009). Sandefur et al. (2011) have shown that algal strains grown in treated wastewater were able to significantly decrease both phosphorus and nitrogen concentrations. When microalgae are grown in piggery wastewater, An et al. (2003) showed that the green alga Botryococcus braunii could remove $80 \%$ of the nitrate content. Another significant component of municipal wastewater is the organic load (Rogers 1996, Fadini et al., 2004). Sewage sourced from human populations is replete with biodegradable compounds such as sugars, amino acids and other breakdown products of digestion (Painter and Viney 1959; Garcia et al. 1991). Although readily mineralized by bacteria during secondary treatment, these organic compounds can also be utilized by mixotrophic algae.

Even though wastewater has great potential as a growth medium for algal-feedstock production, a major caveat is the broad variation in wastewater quality and levels of toxic constituents. Most studies assessing algal growth in wastewater use isolates from lakes, rivers, or 
68 other naturally occurring water bodies (Park et al., 2012; Zhou et al., 2011). Zhou et al. (2011)

69 reported that only a few strains from the genera Chlorella and Scenedesmus have been analyzed

70 for their ability to grow in municipal wastewater. Some work (Liu et al., 2011) has been done to

71 isolate and culture microalgae from wastewater treatment systems, but assessment of their

72 potential use as a biofuel feedstock remains largely unknown. Also, most studies characterizing

73 microalgae for biofuel production typically use axenic strains and sterile media [Price, Yin \&

74 Harrison, 1998; Liu et al., 2011; Zhou et al., 2011), which eliminates the potential role of

75 bacteria in influencing algal growth and lipid production.

76 In order to move forward the prospects of large-scale cultivation of microalgae in

77 wastewater, we set out to assess the potential of microalgae isolated from municipal wastewater

78 treatment systems to serve as candidates for biofuel-feedstock production. The rationale being

79 that microalgae isolated from municipal wastewater would have inherently higher tolerance to

80 wastewater compared to lab strains or isolates from natural systems, as well as an increased

81 capacity to grow mixotrophically to exploit organic compounds in wastewater. The first phase of

82 our assessment is presented in this paper, which focuses on: 1) Isolating algal strains from

83 municipal waste-treatment systems; 2) Characterizing the metabolic growth characteristics of

84 wastewater isolates (i.e., photoautotrophy, mixotrophy, and heterotrophy), including their fatty

85 acid composition; and 3) Assessing the effects of commensal bacteria on algal growth and lipid

86 production compared to axenic strains.

87

88 Materials and Methods

89

Strain Collection and Isolation 
Wastewater samples were collected from three municipal wastewater treatment plants in

91

92

93

94 southern Ontario, Canada including the towns of Port Perry and Whitby, and the city of Hamilton. All municipal wastewater treatment facilities were activated sludge, with the exception of Port Perry, which is a lagoon system of aerated stabilization basins. Wastewater from secondary (i.e., biological) treated effluent was collected in six sterile 1-L Nalgene $\mathrm{TM}^{\mathrm{TM}}$ bottles at mid- and final treatment stages. Wastewater samples were placed into a cooler and stored at $4{ }^{\circ} \mathrm{C}$ until enrichment cultures were initiated within 24-hours of collection. Effluent (100-mL) was placed into sterile Erlenmeyer flasks in triplicate. Flasks were then placed on a cool-fluorescent light bank (12:12 dark/light cycle) to encourage algal growth in whole-effluent. Once growth was confirmed either by naked eye or microscopic examination, 5-mL of the sample was transferred to a $250 \mathrm{~mL}$ Erlenmeyer flask containing either autoclaved BG11 (Rippka et al., 1979) or CHU10 (Stein, 1973) media. Two types of media were chosen in an attempt to isolate a broader diversity of isolates. After growth was established in media, 5-mL of culture was added to new medium and grown for two weeks. This process was repeated two successive times to ensure the compatibility of the algal culture under lab conditions (Anderson, 2005). Isolation from the algal consortium was undertaken by serial dilutions and transferring $100-\mu \mathrm{L}$ of diluted culture to $1.5 \%$ agar for spread plating. Once single colonies were formed they were transferred with the use of a flame sterilized loop to a well in a 24-microwell plate containing 1-mL of either medium. When growth was present and unialgal cultures were confirmed microscopically, the cultures that were unialgal were transferred to fresh media. Morphologically unique strains were selected and maintained for further experimentation. Although several strains of the same genus and sampling location were isolated (Table 1), morphological variations witnessed under the microscope were used to discriminate between 
113 unique strains. Future 18S rRNA sequencing of strains will help to further delineate strain

114 identification. However, past work (Kirkwood et al., 2008) has clearly shown that phylogenetic

115 similarity does not necessarily reflect physiological similarity.

116 Before experimentation, each culture was re-evaluated microscopically to confirm

117 unialgal status. Reference strains Scenedesmus acutus (CPCC 10) and Chlorella kesslerii (CPCC

118 266) were obtained from the Canadian Phycological Culture Collection (CPCC), Waterloo,

119 Ontario, Canada to compare their growth characteristics to wastewater strains. A comparison

120 between CHU10 and BG11 media revealed that after 5-10 days of growth, algal strains grown in

121 CHU10 started to yellow while BG11 strains retained a healthy green colour. As such, all

122 subsequent cultures and growth experiments were conducted with BG11. The nitrogen

123 concentration in BG11 was reduced by a factor of ten to bring it down to a level more closely

124 aligned with wastewater concentrations.

125 Experimental Growth Conditions

126 All algal growth experiments were conducted in an Algaetron ${ }^{\mathrm{TM}}$ environmental growth

127 chamber (Algaetron Photon System Instruments, Czech Republic) with a built in shaker table.

128 Growth parameters were set to $200 \mathrm{rpm}$ and $150 \mu \mathrm{mol}$ photons $\mathrm{m}^{-2} \mathrm{~s}^{-1}$. A temperature of $22{ }^{\circ} \mathrm{C}$

129 was used to match the ambient temperature in the lab to minimize any variations encountered

130 when sampling. To assess the effect of growth condition (photoautotrophy, mixotrophy,

131 heterotrophy) on growth rate and fatty acid accumulation, all three growth conditions were tested

132 concurrently for each strain. For the photoautotrophic conditions, all organics were removed

133 from the BG11 media (citric acid, ferric ammonium citrate, and sodium EDTA) and the iron was

134 replaced with ferric chloride similar to the method described by Kirkwood, Fulthorpe \&

135 Nalewajko (2003). The mixotrophic conditions utilized the organic-free medium from the 
136 photoautotrophic trials with the addition of either glucose $(14 \mathrm{mM})$ or acetate $(3 \mathrm{mM})$, similar to

137 Park et al (2012) and Kirkwood, Fulthorpe \& Nalewajko (2003). The heterotrophic growth trials

138 utilized the same medium as the mixotrophic trials with the exception of being placed in a

139 cardboard box to exclude illumination. Cultures for experimentation were grown in 50-mL

140 Erlenmeyer flasks in triplicate for seven days. To standardize inocula among experiments and

141 strains, mid-exponential phase inocula with similar cell densities were used. Standard curves of

142 cell density for each algal strain were based on cell counts using a haemocytometer and optical

143 density measured with a Genesys 10S UV-Vis Spectrophotometer (ThermoScientific, Waltham,

144 Massachusetts). Growth rates for each algal strain were calculated from the slope of log-

145 transformed 7-day growth curves $\left(\mathrm{d}^{-1}\right)$.

146 Antibiotic Treatment of Algal Isolates

147 In order to measure fatty acids derived only from the algal isolates, all strains were

148 subjected to a series of antibiotic treatments to remove commensal bacteria. This was

149 accomplished through the application of streptomycin and penicillin following the methods

150 outlined by Droop (1967). To verify axenic status after 24 hours of incubation in the antibiotic

151 media, a 1-mL aliquot of each dilution was transferred to $1.5 \%$ agar containing peptone via pour

152 plate method. The plates were then placed on the light table and monitored over the course of 48

153 hours for bacterial growth. If no growth was visible on the plates, microscopic examination was

154 performed to verify axenic status. If a sampled failed to achieve axenic status the procedure was

155 repeated. Strains were classified as non-axenic if either the antibiotics proved ineffective at

156 eliminating the bacteria, or the antibiotics prevented algal growth. Parent, non-axenic cultures of

157 all axenic strains were maintained for experiments comparing fatty acid profiles and neutral lipid 158 concentrations. 
Fatty Acid Methyl Esters (FAME) Analysis

Due to the extremely low biomass yields for all algal strains grown under heterotrophic conditions, only photoautotrophic and mixotrophic cultures were analysed for fatty acid methyl esters (FAME). Both axenic and non-axenic forms of the same strain were included in FAME analysis. The lipid extraction procedure was based on the FAME synthesis described in O'fallon et al. (2007). In brief, 7-mL subsamples of microalgae with a cellular density $\geq 10^{5}$ cells $\cdot \mathrm{mL}^{-1}$ was collected at day seven of the experiment. These samples were centrifuged at $1690 \mathrm{~g}$ force for 5 minutes. The supernatant was discarded and the samples were stored in $-20{ }^{\circ} \mathrm{C}$ until freeze dried. Once freeze dried (typically $24-48$ hours) samples could be processed. The fatty acids derived via the lipid extraction method was determined by capillary Agilent J\&W GC column $(30 \mathrm{~m} \cdot 0.25 \mathrm{~mm} \cdot 0.25 \mu \mathrm{m})$ installed in a Varian 450 gas chromatograph. The initial oven temperature was $135^{\circ} \mathrm{C}$ held for $4 \mathrm{~min}$, followed by an increase to $250{ }^{\circ} \mathrm{C}$ at a rate of $4{ }^{\circ} \mathrm{C} \cdot \mathrm{min}^{-}$ , followed by a hold at this temperature for $10 \mathrm{~min}$. The front injector was $250{ }^{\circ} \mathrm{C}$ and held for $20 \mathrm{~min}$, the middle was $110{ }^{\circ} \mathrm{C}$ for $1 \mathrm{~min}$, and the rear injector was held at $180{ }^{\circ} \mathrm{C}$ for $20 \mathrm{~min}$. A mixture of Helium and Nitrogen was used as the carrier gas with a split ratio of 10:1 at a flow rate of $1-\mathrm{mL} \cdot \mathrm{min}^{-1}$ for $3.5 \mathrm{~min}$ then shut off. The retention times of each sample were analyzed using an electron impact ion trap mass spectrometer. The standards used for analysis were carbon chain 8 to carbon chain 24 (C8-C24) along with a Bacterial Acid Methyl Ester (BAME) fatty acid mix. The BAME standard was used to filter out non-algal fatty acids. Fatty acids were identified through the use of MS workstation by comparing their retention times to known fatty acid methyl standards. Each chromatograph was then analyzed manually and any peaks that were above the background noise threshold were library searched to determine if the peak was an associated fatty acid. All standards were purchased from Sigma Aldrich (Canada). 
182

183

184

185

186

187

188

189

190

191

192

193

194

195

196

197

198

199

200

201

202

203

204

\section{Quantification of Neutral Lipids}

Similar to the FAME analyses, only photoautotrophic and mixotrophic cultures were

analysed for neutral lipids. Both axenic and non-axenic forms of select strains were included in neutral lipids analysis. Neutral lipids in algal cells were determined using the standard Nile Red assay. A calibration curve for neutral lipids was based on the fluorescence response of varying triolein concentrations. Subsamples $(1.5-\mathrm{mL})$ of algal culture from the growth experiments were stored at $-20{ }^{\circ} \mathrm{C}$ prior to analysis. Due to the limited filter set available, we decided to use an excitation of 590 and emission of 640 nanometers based on the strong fluorescent response of the Nile Red dye at these wavelengths. Nile Red was dissolved in HPLC grade acetone at a concentration of $500 \mu \mathrm{g} \cdot \mathrm{mL}^{-1}$, and was stored in complete darkness in a vial covered in aluminium foil to prevent photodegradation. In a black-opaque 96 microwell plate, $150-\mu \mathrm{L}$ of algal sample was aliquoted into the wells in triplicate along with triplicate blanks. Following the addition of the algal samples to the wells, $90-\mu \mathrm{L}$ of Nile Red was added to the treatment wells to dye the algal cells. The microwell plate was covered and incubated in darkness at room temperature for 10 minutes to allow dye penetration into the cells. After incubation, the plate was immediately analyzed with a Synergy HT multimode plate reader (BioTEK, Winooski, Vermont).

\section{Data Analysis}

To determine statistically significant differences between growth condition treatments and strains, Sigma Plot 12 was used to run Analysis of Variance (ANOVA) on exponential growth rates and neutral lipid concentrations. A Shapiro-Wilk test was performed to determine if the data were normally distributed and an All Pairwise Multiple Comparison (Holm-Sidak method) was performed to detect significant differences between individual strains. The 
205 Paleontological Statistics (PAST) software package (v3) (Hammer, Harper \& Ryan, 2001) was

206 used for cluster analysis and Principal Component Analysis (PCA). Cluster analysis was based

207 on the average growth rate of each of 19 strains under each of 5 growth conditions

208 (photoautotrophy, mixotrophy-glucose, mixotrophy-acetate, heterotrophy-glucose, heterotrophy-

209 acetate). PCA was based on the percent abundance of fatty acids identified in the FAME

210 analysis, and least-squares linear regression was used to regress PCA axis scores against

211 individual fatty acids.

212

213

Results

214

Microalgae were successfully isolated from all wastewater treatment plants. Table 1

215 provides a list of strains, media used to isolate each strain, and the original sampling location.

216 Most of the 19 wastewater strains isolated were chlorophyte microalgae, dominated by

217 Scenedesmus and Chlorella species. The only non-chlorophyte taxon isolated was the

218 cyanobacterium Microcystis. With respect to the diversity of taxa isolated, Port Perry wastewater

219 treatment plant provided strains from all isolated genera, followed by Hamilton and Whitby

220 wastewater treatment plants. The application of antibiotic treatment rendered nine of the

221 wastewater isolates axenic (Table 1).

222 Average growth rates $(\mathrm{n}=3)$ for each strain grown under photoautotrophic, mixotrophic

223 and heterotrophic conditions are presented in Figure 1. When comparing the CPCC strains to

224 their taxonomically-similar counterparts from wastewater systems, most Chlorella wastewater

225 isolates (with the exception of $\mathrm{C} 3 \mathrm{~N}$ ) grew significantly faster (ANOVA and Holm-Sidak,

$226 \mathrm{p}<0.05)$ than the $\mathrm{CPCC}$ reference strain $\mathrm{C} 1 \mathrm{U}$, particularly under mixotrophic growth conditions.

227 In contrast, there were only a few Scenedesmus wastewater strains that grew significantly 
228 (ANOVA, $\mathrm{p}<0.05$ ) faster under mixotrophic conditions with glucose, compared to the CPCC

229 reference strain S1B. In general, the Scenedesmus strains grew significantly slower (ANOVA,

$230 \mathrm{p}<0.05)$ than the Chlorella strains. The fastest growth rates for any strain were under

231 mixotrophic conditions. One other comparably fast growing strain was D1N, which grew well

232 heterotrophically on acetate. Although several strains could grow fairly well on glucose under

233 heterotrophic conditions, including D1N and most of the Chlorella strains, most could not grow

234 very well on either glucose or acetate under heterotrophic conditions.

235 To assess the trophic similarities of strains based on photoautotrophic, mixotrophic, and

236 heterotrophic growth, cluster analysis was performed (Figure 2). Although the cophenetic

237 correlation coefficient (0.73) indicates that the hierarchical structure in the dendrogram

238 represents the actual similarity moderately well, most dendrogram nodes had weak to moderate

239 agreement based on bootstrap values (1000 replicates). The dendrogram reveals some taxonomic

240 clustering, but mostly a diversity of functional growth-types. The most physiologically similar

241 strains belong to different taxonomic genera, such as Chlorella $\mathrm{C} 3 \mathrm{~N}$ and Botrydiopsis B1N. A

242 few strains were less than $80 \%$ similar to all the other strains, including Scenedesmus $\mathrm{S} 7 \mathrm{H}$ and

243 Chlorella C4C.

$244 \quad$ Fatty acid profiles via FAME analysis were determined for each strain under

245 photoautotrophic and mixotrophic conditions with glucose or acetate (Figure 3A-C). To improve

246 clarity, strains were removed from the analysis if they had less than $1 \%$ fatty acids detected.

247 Under photoautotrophic conditions (Figure 3A), most non-axenic strains remained in the analysis

248 with detectable fatty acids. Remaining axenic strains tended to cluster to the left of the y-axis,

249 indicating their profiles were different than many non-axenic strains. Although some clustering

250 of strains occurred, most strains were fairly unique in their fatty acid profiles, either being 
251 dominated by C 17 or C 23 fatty acids. When strains were grown under mixotrophic conditions

252 with acetate (Figure 3B), the number of fatty acids detected were somewhat comparable to

253 photoautotrophic conditions (Figure 3A), but with a few fatty acid substitutions including the

254 addition of arachidic acid and the absence of 12-methyltetradecanoic acid and 13-

255 methyltetradecanoic acid. More strains had detectable fatty acids under mixotrophy with acetate,

256 particularly non-axenic strains. Growth with acetate clearly changed the fatty acid composition

257 of strains compared to photoautotrophic growth. When the strains were grown with glucose, a

258 comparatively larger array of fatty acids were detected, but in low concentrations (Figure 3C).

259 More strains had detectable fatty acids than the photoautotrophy treatment, and more strains

260 clustered together indicating a high degree of similarity. The strains S4N and C5C were found to

261 have very distinct fatty acid profiles based on their location on the biplot.. Table 2 provides a

262 summary of the most important fatty acids driving fatty acid profiles under the different growth

263 conditions. As visualized in the biplots, linoleic, oleic, and palmitic acids were dominant fatty

264 acids under all growth conditions (Table 2). Under mixotrophic growth with glucose, the number

265 of important fatty acids explaining the variation in fatty acid profiles doubles in comparison to

266 photoautotrophic growth and mixotrophic growth with acetate.

The total neutral lipids for matched axenic and non-axenic algal strains grown under

268 photoautotrophic and mixotrophic conditions are presented in Table 3. Overall, it is apparent that

269 photoautotrophy favours the production of total neutral lipids, although strain B1N was the

270 exception where mixotrophy on glucose produced significantly more neutral lipids. Notable

271 differences between axenic and non-axenic strains were found for all growth conditions. Axenic

272 strains $\mathrm{C}_{3} \mathrm{~N}^{*}$ and $\mathrm{S} 7 \mathrm{H}^{*}$ had approximately an order of magnitude higher total neutral lipids than 
273 other algal strains. Strain C3N in particular was a high neutral-lipid producer compared to all of

274 the strains, regardless of axenic status.

275

276 Discussion

277

The chlorophyte genera isolated from municipal wastewater treatment plants in this

278

279

280

281

282

283

284

285

286

287

288

289

290

291

292

293

294

295 study: Chlorella, Botrydiopsis, Dictyochloris, Ellipsoidon and Scenedesmus reflect a variety of algal strains that are viable in wastewater, but also easy to grow under laboratory conditions. Although found to be among the fastest growing strains, the cyanobacterium Microcystis was not a notable producer of fatty acids in this study. Chlorella strains were prevalent across the three wastewater treatment systems. This may be due in part to Chlorella's high tolerance of low oxygen conditions, such as in wastewater treatment systems (Shanthala, Hosmani \& Hosetti, 2009). Park et al. (2012) commented that both Scenedesmus and Chlorella are among the most commonly isolated species from wastewater treatment effluent, which explains their high prevalence in all sampling locations in this study.

Mixotrophic growth, with either glucose or acetate, resulted in higher growth rates compared to photoautotrophic growth for the large majority of strains. This was not unexpected as others have demonstrated higher growth yields for algae under mixotrophic growth conditions (Kirkwood, Fulthorpe \& Nalewajko, 2003; Das, Aziz \& Obbard, 2011; Yan et al., 2012). Yan et al. (2012) also demonstrated that the addition of either glucose or acetate actually increased the energy conversion efficiencies over photoautrophic growth. In most cases under heterotrophic growth, the initial algal inoculation either resulted in cell death or showed minimal to no growth. Price, Yin \& Harrison (1998) found that many microalgal taxa derived from naturally occurring water bodies have generally adapted their cellular processes to daily light fluctuations and 
296 therefore are unable to grow strictly heterotrophically. As such, mixotrophy rather than

297 photoautotrophy or heterotrophy appears to be the ideal strategy for maximizing growth rate and

298 biomass yield. Since municipal wastewater and some industrial wastewaters (e.g. pulp and paper

299 effluent) are replete with organic compounds, including sugars, amino acids, and other

300 degradation products, it would be an ideal medium for supplementing algal growth.

301 Similar to other studies (e.g., Nascimento et al., 2013), the fastest growing strains were

302 not necessarily the best lipid producers (compare Figure 1 and Table 3 ). Also, the relative

303 amount of fatty acids produced in each strain varied by axenic status and growth condition and in

304 general, the highest amount of biofuel-targeted fatty acids, such as oleic and linoleic FAMEs,

305 were produced by non-axenic algal strains, particularly slower growing Scenedesmus isolates

306 grown mixotrophically with glucose (Figure 5). Under photoautotrophic conditions, any bacterial

307 effect on fatty acid profiles of non-axenic microalgal strains were not discernable. However, the

308 effect became evident under mixotrophic growth, particularly with glucose. A possible

309 explanation is that the bacteria consumed not only the organic compounds in the microalgal

310 cultures, but inorganic nutrients as well. This would create nutrient-deprived conditions for the

311 host microalgal strain, which in turn could induce lipid accumulation as a stress response.

Increased lipid production in algae is a common response to stressful or rapidly changing

conditions (Saha et al., 2013). Since the relative amount and type of fatty acids increased most

notably in non-axenic strains of microalgae in our study, this may be the result of a nutrient-

stress response via competition with bacteria. Liu et al. (2012) discovered that when glucose was

added to growth media, it stimulated bacterial growth in algal cultures, which in turn reduced the

317 availability of inorganic nutrients to the algae. A similar result was found when Scenedesmus 
319 became limited at the plateau of the alga's growth cycle (Daufresne et al., 2008). When grown in 320 co-culture, bacteria can also produce allelopathic exudates that either inhibit algal growth or lyse 321 cells (Mayali \& Azam, 2004). However, it is unclear if bacterial allelopathy can also induce

322 hyperaccumulation of lipids in algae.

Although for the majority of strains, the total amount of neutral lipids was comparable between axenic and non-axenic strains, there were two striking exceptions (Table 3). Strains $\mathrm{C} 3 \mathrm{~N}$ and $\mathrm{S} 7 \mathrm{H}$ accumulated more neutral lipids particularly under axenic and photoautotrophic conditions. If hyperaccumulation of lipids tends to occur under stressful growth conditions, the

327

328

results for these two strains may indicate that photoautotrophic growth without bacteria is a stressful growth condition. Of course, this is a preliminary finding, but does highlight the unique differences that can exist among algal taxa isolated from the same environment. Regardless of mechanism, these findings also highlight that, depending on the strain you are working with, the presence of bacteria can have significant impacts on not only the type of fatty acids being produced, but the total amount.

Comparing the microalgal strains under the three different growth conditions showed that axenic strains had a small but detectable difference in fatty acid composition compared to their non-axenic counterparts. A similar result was noted with $C$. vulgaris and C. sorokiniana when grown with the bacterium $A$. brasilene, which caused the variation in fatty acids to change from five to eight different fatty acids with increasing amounts of unsaturation (de Bashan et al., 2002). Adding glucose to media can change the lipid composition in photoautotrophically grown microalgae by significantly increasing oleic acid concentration (Sunja et al., 2011). This was also found in our experiments, where an increased number of strains produced oleic acid under mixotrophic growth with glucose compared to photoautotrophic growth. Additionally, Cherisilp 
342 and Torpee (2012) have confirmed that microalgae grown under mixotrophic conditions using

343 glucose showed an overall increase in lipid content over photoautotrophic growth and

344 heterotrophic growth, which we also found.

345 Overall, our assessment of wastewater microalgae has not only shown that mixotrophy

346 can significantly increase growth rate, but that most wastewater Chorella and Scenedesmus

347 strains exceed the mixotrophic growth-rates of representative culture collection strains. This

348 infers that wastewater strains may be ideal candidates for growth in organic-substrate rich

349 wastewater for biomass and/or biofuel feedstock production. Most of the Chlorella wastewater

350 strains were the fastest growers, particularly under mixotrophic and heterotrophic growth

351 conditions. This may increase their potential as biofuel feedstock due to their growth-condition

352 versatility. Additionally, the Chlorella wastewater isolates from this study produced total-lipid

353 concentrations on par with wastewater-grown strains (as total lipid in culture-medium) in a

354 study by Woertz et al (2009).

355 Although fast growth is an ideal characteristic for any algal strain used in biofuel

356 feedstock production, it must be coupled with comparatively high lipid yields. As previously

357 discussed, the fastest growing microalgal strains were typically not the highest lipid producers.

358 Thus acceptable trade-offs in growth rate vs. lipid yield must be established. To date, the

359 majority of research in algal biofuels involves the maintenance of axenic or low bacterial

360 contamination in microalgal cultures. Yet, our study has demonstrated that the fatty acid amount

361 and composition can change with bacterial presence. Additionally, the even blend of saturated

362 and unsaturated fatty acids tended to be found in non-axenic microalgal cultures. These findings

363 bode well for growing microalgae in bacteria-laden wastewater. However, it remains to be

364 determined if microalgal growth in wastewater with bacteria results in higher lipid yields and 
365 desirable fatty acid composition. As such, the next phase of our research is focusing on the

366 efficacy of growing microalgal wastewater-isolates in municipal wastewater, and assessing the 367 conditions under which growth and lipid production are optimized.

368

369 Acknowledgements

370 The authors wish to thank Heloise Breton and Jenna Comstock for assistance with the

371 FAME protocol and GC-MS calibration and set-up. 


\section{References}

373 An JY, Sim SJ, Lee JS, Kim BW (2003) Hydrocarbon production from secondarily treated

374 piggery wastewater by the green alga Botryococcus braunii. J Appl Phycol 15:185-191.

375 Anderson RA (2005) Algal culturing techniques. Elsevier Science and Technology Books, $376 \quad$ Academic Press, London, UK

377 Brennan L, Owende P (2010) Biofuels from microalgae - A review of technologies for 378 production, processing, and extractions of biofuels and co-products. Renew Sustain Energy Rev 14:557-577.

Cherisilp B, Torpee S (2012) Enhanced growth and lipid production of microalgae under mixotrophic culture condition: Effect of light intensity, glucose concentration and fedbatch cultivation, Bioresour Technol 110:510-516.

Chinnasamy S, Bhatnagar A, Claxton R, Das KC (2010) Biomass and bioenergy production of microalgae consortium in open and closed bioreactors using untreated carpet industry effluent as growth medium. Bioresour Technol 101:6751-6760.

Cho SH, Ji SC, Hur SB, Bae J, Park IS, Song YC (2007) Optimum temperature and salinity conditions for growth of green algae Chlorella ellipsoidea and Nannochloris oculata. Fish

da Silva TL, Reis A, Medeiros R, Oliveira AC, Gouveia L (2009) Oil production towards biofuel from autotrophic microalgae semicontinuous cultivations monitorized by flow cytometry. Appl Biochem Biotechnol 159:568-578. 
394 Daufresne T, Lacroix G, Benhaim D, Loreau M (2008) Coexistence of algae and bacteria: a test 395 of the carbon hypothesis. Aquat Microb Ecol 53:323-332.

396 de Bashan LE, Bashan Y, Moreno M, Lebsky VK, Bustillos JJ (2002) Increased pigment and 397 lipid content, lipid variety, and cell and population size of the microalgae Chlorella spp. 398 when co-immobilized in alginate beads with the microalgae-growth-promoting bacterium $399 \quad$ Azospirillum brasilense. Can J Microbiol 48:514-521.

400 de la Noue J, Laliberte G, Proulx D (1992) Algae and waste water. J Appl Phycol. 4:247-254. 401 Droop MR (1967) A procedure for routine purification of algal cultures with antibiotics. Br 402 Phycol Bull 3:295-297.

403

404

405

406

407

408

409

410

411

412

413

414

Fadini PS, Jardim WF, Guimarães JR (2004) Evaluation of organic load measurement techniques in a sewage and waste stabilisation pond. J Brazil Chem Soc 15:131-135

García C, Hernández T, Costa F (1991) Study on water extract of sewage sludge composts. Soil Sci Plant Nut 37:399-408.

Hammer Ø., Harper DAT, Ryan PD (2001) PAST: Paleontological Statistics software package for education and data analysis. Paleontol Electron 4:9.

Kirkwood AE, Buchheim JA, Buchheim MA, Henley WJ (2008) Cyanobacterial diversity and halotolerance in a variable hypersaline environment. Microb Ecol 55:453-465.

Kirkwood AE, Nalewajko C, Fulthorpe RR (2003) Physiological characteristics of cyanobacteria in pulp and paper waste-treatment systems. J Appl Phycol 15:325-335.

Lau PS, Tam NFY, Wong YS (1995) Effect of algal density on nutrient removal from primary settled wastewater. Environ Pollut 89:59-66. 
415 Liu J, Huang JC, Sun Z, Zhong YJ, Jiang Y, Chen F (2011) Differential lipid and fatty acid 416 profiles of photoautotrophic and heterotrophic Chlorella zofingiensis: Assessment of algal oils for biodiesel production. Bioresour Technol 102:106-110.

418

419

420

421

422

423

424

425

426

427

428

429

430

431

432

433

434

435

436

Liu H, Zhou Y, Xiao W, Ji L, Cao X, Song C (2012) Shifting nutrient-mediated interactions between algae and bacteria in a microcosm: Evidence from alkaline phosphatase assay. Microbiol Res 167:292-298.

Mayali X, Azam F (2004) Algicidal Bacteria in the Sea and their Impact on Algal Blooms. J Eukaryot Microbiol 51:139-144.

Mehanna M, Saito T, Yan J, Hickner M, Cao X, Huang X, Logan BE (2010) Using microbial desalination cells to reduce water salinity prior to reverse osmosis. Energy Environ Sci 3:1114-1120.

Nascimento IA, Marques SSI, Cabanelas ITD, Pereira SA, Druzian JI, de Souza CO, Nascimento MA (2013) Screening microalgae strains for biodiesel production: lipid productivity and estimation of fuel quality based on fatty acid profiles as selective criteria. BioEnerg Res 6 $1-13$.

O'fallon JV, Busboom JR, Nelson ML, Gaskins CT (2007) A direct method for fatty acid methyl ester synthesis: application to wet meat tissues, oils, and feedstuffs. J Animal Sci 85:15111521.

Painter, H.A. and Viney, M., 1959. Composition of a domestic sewage. Journal of Biochemical and Microbiological Technology and Engineering, 1(2), pp.143-162.

Park KC, Whitney C, McNichol JC, Dickinson KE, MacQuarrie S, Skrupski BP, Zou J, Wilson KE, O’Leary SJB, McGinn PJ (2012) Mixotrophic and photoautotrophic cultivation of 14 
437

438

439

440

441

442

443

444

445

446

447

448

449

450

451

452

453

454

455

456

457

458

microalgae isolates from Saskatchewan, Canada: potential applications for wastewater remediation for biofuel production. J Appl Phycol 24:339-348.

Price LL, Yin K, Harrison PJ (1998) Influence of continuous light and L:D cycles on the growth and chemical composition of Prymnesiophyceae including coccolithophores. J Exp Mar Biol Ecol 223:223-234.

Rippka R, Deruelles J, Waterbury J, Herdman M, Stanier R (1979) Generic assignments, strain histories and properties of pure cultures of cyanobacteria. J Gen Microbiol 111:1-61.

Roddy DJ (2013) Biomass in a petrochemical world. Interface Focus 3:1-8.

Rogers HR (1996) Sources, behaviour and fate of organic contaminants during sewage treatment and in sewage sludges. Sci Total Environ 185:3-26.

Saha SK, McHugh E, Hayes J, Moane S, Walsh D, Murray P (2013) Effect of various stressregulatory factors on biomass and lipid production in microalga Haematococcus pluvialis. Bioresour Technol 128 118-124.

Sandefur HN, Matlock MD, Costello TA (2011) Seasonal productivity of periphytic algal community for biofuel feedstock generation and nutrient treatment. Ecol Eng 37:14761480.

Sandstrom K (1995) Modeling the effects of rainfall variability on groundwater recharge in semi-arid Tanzania. Nord Hydrol 26:313-330.

Shanthala M, Hosmani SP, Hosetti BB (2009) Diversity of phytoplankton in waste stabilization pond at Shimoga Town, Karnataka State, India. Environ Monit Assess 151:437-443.

Stein J (1973) Handbook of Phycological methods. Culture methods and growth measurements, Cambridge University Press, Cambridge, UK 
459 Sunja C, Lee D, Luong TT, Park S, Oh YK, Lee T (2011) Effects of carbon and nitrogen sources

460 on fatty acid contents and composition in the green microalga Chlorella sp. 227. J

$461 \quad$ Microbiol Biotechnol 21:1073-1080.

462 Tsukahara K, Sawayama S (2005) Liquid fuel production using microalgae. J Jpn Pet Inst $463 \quad 48: 251-259$.

464 Woertz I, Feffer A, Lundquist T, Nelson Y (2009) Algae grown on dairy and municipal 465 wastewater for simultaneous nutrient removal and lipid production for biofuel feedstock. J $466 \quad$ Environ Eng 135:1115-1122.

467 Yan R, Zhu D, Zhang Z, Zeng Q, Chu J (2012) Carbon metabolism and energy conversion of 468 Synechococcus sp. PCC 7942 under mixotrophic conditions: comparison with 469 photoautrophic condition. J Appl Phycol 24:657-668.

470 Zhou W, Li Y, Min M, Hu B, Chen P, Ruan R (2011) Local bioprospecting for high-lipid 471 producing microalgal strains to be grown on concentrated municipal wastewater for biofuel $472 \quad$ production. Bioresour Technol 102:6909-6919. 


\section{Table $\mathbf{1}$ (on next page)}

List of microalgal strains.

List of microalgal strains assessed in this study including nineteen municipal wastewater treatment plant (WWTP) isolates and two Canadian Phycological Culture Collection (CPCC) reference strains. 
Table 1: List of microalgal strains assessed in this study including nineteen municipal wastewater treatment plant (WWTP) isolates and two Canadian Phycological Culture Collection (CPCC) reference strains.

\begin{tabular}{|c|c|c|c|c|}
\hline Taxonomic ID & Strain ID & Isolating Medium & Treatment stage & Site of Origin \\
\hline Botrydiopsis & $\mathrm{B} 1 \mathrm{~N}$ & CHU10 & Mid-stage secondary treatment & Port Perry, Ontario WWTP \\
\hline Botrydiopsis & $\mathrm{B} 2 \mathrm{H}$ & BG11 & Final stage of secondary treatment & Hamilton, Ontario WWTP \\
\hline Chlorella kesslirii & $\mathrm{C} 1 \mathrm{U}$ & Unknown & CPCC Reference strain (CPCC 266) & Unknown (USA) \\
\hline Chlorella & $\mathrm{C} 3 \mathrm{~N}$ & CHU10 & Mid-stage secondary treatment & Port Perry, Ontario WWTP \\
\hline Chlorella & $\mathrm{C} 4 \mathrm{C}$ & CHU10 & Mid-stage secondary treatment & Whitby, Ontario WWTP \\
\hline Chlorella & $\mathrm{C} 5 \mathrm{C}$ & CHU10 & Mid-stage secondary treatment & Whitby, Ontario WWTP \\
\hline Chlorella & $\mathrm{C} 6 \mathrm{C}$ & CHU10 & Mid-stage secondary treatment & Whitby, Ontario WWTP \\
\hline Dictyochloris & $\mathrm{D} 1 \mathrm{~N}$ & CHU10 & Final stage of secondary treatment & Port Perry, Ontario WWTP \\
\hline Ellipsoidon & $\mathrm{E} 1 \mathrm{H}$ & BG11 & Final stage of secondary treatment & Hamilton, Ontario WWTP \\
\hline Ellipsoidon & $\mathrm{E} 2 \mathrm{C}$ & CHU10 & Mid-stage secondary treatment & Whitby, Ontario WWTP \\
\hline Ellipsoidon & $\mathrm{E} 3 \mathrm{~N}$ & CHU10 & Final stage of secondary treatment & Port Perry, Ontario WWTP \\
\hline Microcystis & M1H & BG11 & Mid-stage secondary treatment & Hamilton, Ontario WWTP \\
\hline Scenedesmus acutus & S1B & Unknown & CPCC Reference strain CPCC 10 & Boucher Lake, ON \\
\hline Scenedesmus & $\mathrm{S} 2 \mathrm{~N}$ & CHU10 & Final stage of secondary treatment & Port Perry, Ontario WWTP \\
\hline Scenedesmus & $\mathrm{S} 3 \mathrm{~N}$ & CHU10 & Final stage of secondary treatment & Port Perry, Ontario WWTP \\
\hline Scenedesmus & S4N & CHU10 & Mid-stage secondary treatment & Port Perry, Ontario WWTP \\
\hline Scenedesmus & $\mathrm{S} 5 \mathrm{~N}$ & CHU10 & Final stage of secondary treatment & Port Perry, Ontario WWTP \\
\hline Scenedesmus & $\mathrm{S} 6 \mathrm{H}$ & BG11 & Mid-stage secondary treatment & Hamilton, Ontario WWTP \\
\hline Scenedesmus & S7H & BG11 & Mid-stage secondary treatment & Hamilton, Ontario WWTP \\
\hline Scenedesmus & $\mathrm{S} 8 \mathrm{C}$ & CHU10 & Mid-stage secondary treatment & Whitby, Ontario WWTP \\
\hline Scenedesmus & $\mathrm{S} 9 \mathrm{C}$ & CHU10 & Mid-stage secondary treatment & Whitby, Ontario WWTP \\
\hline
\end{tabular}




\section{Table 2 (on next page)}

Regressions using PCA scores

Regression coefficients (R2) from least-squares linear regression of principle component axis scores and fatty acid proportions for photoautotrophic and mixotrophic conditions. Only statistically significant coefficients are reported $(p \leq 0.05)$. 
Table 2: Regression coefficients $\left(\mathrm{R}^{2}\right)$ from least-squares linear regression of principle component axis scores and fatty acid proportions for photoautotrophic and mixotrophic conditions. Only statistically significant coefficients are reported ( $\mathrm{p} \leq 0.05)$.

\begin{tabular}{lcc}
\hline Fatty Acids & $\begin{array}{c}\text { PC Axis 1 } \\
\left(\mathbf{R}^{2}\right)\end{array}$ & $\begin{array}{c}\text { PC Axis 2 } \\
\left(\mathbf{R}^{2}\right)\end{array}$ \\
\hline $\begin{array}{l}\text { Photoautotrophy } \\
\text { linoleic acid methyl ester }\end{array}$ & \\
oleic acid methyl ester (cis) & 0.8 & \\
palmitic acid methyl ester & 0.7 & \\
12-methyltetradecanoic acid methyl ester & 0.92 & 0.72 \\
erucic acid methyl ester & & 0.86 \\
\hline Mixotrophy-Acetate & & \\
linoleic acid methyl ester & 0.76 & \\
oleic acid methyl ester (cis) & 0.87 & \\
palmitic acid methyl ester & 0.81 & \\
arachidic acid methyl ester & & 0.76 \\
14-methylpentadecanoic acid methyl ester & & 0.74 \\
\hline Mixotrophy-Glucose & & \\
arachidic acid methyl ester & 0.94 & \\
linoleic acid methyl ester & 0.88 & \\
myristic acid methyl ester & 0.96 & \\
oleic acid methyl ester (cis) & 0.95 & \\
palmitic acid methyl ester & 0.98 & \\
palmitoleic acid methyl ester & 0.94 & \\
stearic acid methyl ester & 0.96 & \\
13-methyltetradecanoic acid methyl ester & & 0.94 \\
14-methylpentadecanoic acid methyl ester & & 0.93 \\
15-methylhexadecanoic acid methyl ester & & 0.94 \\
9,10-methylene-hexadecanoic acid ME & & \\
margaric acid methyl ester & & \\
pentadecanoic acid methyl ester & & 0.94 \\
& & \\
\end{tabular}




\section{Table 3 (on next page)}

Neutral lipids in isolates under different trophic conditions.

Comparison of mean $(n=3)$ total neutral lipids among select axenic and non-axenic strains (axenic strains denoted by * beside the strain name). Standard deviations are in brackets. All treatments for each strain were compared using ANOVA $(\alpha=0.05)$ and the Holm-Sidak method. Statistically significant differences are denoted by: $A=$ photoautotrophic is significantly different from mixotrophic (glucose); $B=$ photoautotrophic is significantly different from mixotrophic (acetate); $C=$ mixotrophic (glucose) is significantly different from mixotrophic (acetate); $Z$ = all treatments are significantly different from each other. 
Table 3. Comparison of mean $(n=3)$ total neutral lipids among select axenic and non-axenic strains (axenic strains denoted by * beside the strain name). Standard deviations are in brackets. All treatments for each strain were compared using ANOVA ( $\alpha$ $=0.05$ ) and the Holm-Sidak method. Statistically significant differences are denoted by: $\mathrm{A}=$ photoautotrophic is significantly different from mixotrophic (glucose); $\mathrm{B}=$ photoautotrophic is significantly different from mixotrophic (acetate); $\mathrm{C}=$ mixotrophic (glucose) is significantly different from mixotrophic (acetate); $\mathrm{Z}=$ all treatments are significantly different from each other.

\begin{tabular}{|c|c|c|c|c|}
\hline \multirow[b]{3}{*}{ Strain ID } & \multicolumn{4}{|c|}{ Neutral Lipids $\left(\mathrm{ng} \cdot\right.$ cell $\left.^{-1}\right)$} \\
\hline & & & & Multiple Comparisons \\
\hline & Photoautotrophic & Mixotrophic (Glucose) & Mixotrophic (Acetate) & Statistical Differences \\
\hline $\mathrm{B} 1 \mathrm{~N}^{*}$ & $22.1(0.56)$ & $45.9(0.256)$ & $14.9(0.186)$ & $\mathrm{Z}$ \\
\hline $\mathrm{B} 1 \mathrm{~N}$ & $7.49(0.334)$ & $62.6(0.359)$ & $5.06(0.012)$ & Z \\
\hline $\mathrm{C} 3 \mathrm{~N}^{*}$ & $776(24.9)$ & $565(15.88)$ & $714(16.1)$ & Z \\
\hline $\mathrm{C} 3 \mathrm{~N}$ & $318(18.2)$ & $262(4.238)$ & $172(2.18)$ & $\mathrm{Z}$ \\
\hline D1N* & $25.6(0.396)$ & $5.22(0.046)$ & $4.84(0.07)$ & $\mathrm{AB}$ \\
\hline $\mathrm{D} 1 \mathrm{~N}$ & $34.2(2.09)$ & $5.75(0.060)$ & $5.59(0.181)$ & $\mathrm{AB}$ \\
\hline $\mathrm{S} 2 \mathrm{~N}^{*}$ & $59.6(2.16)$ & $30.5(0.293)$ & $26.4(0.451)$ & $\mathrm{AB}$ \\
\hline $\mathrm{S} 2 \mathrm{~N}$ & $45.6(2.35)$ & $44.4(0.269)$ & $34.5(0.913)$ & $\mathrm{BC}$ \\
\hline $\mathrm{S} 3 \mathrm{~N}^{*}$ & $49.1(0.41)$ & $36.4(1.315)$ & $12.6(0.154)$ & $\mathrm{Z}$ \\
\hline S3N & $70.5(3.19)$ & $43.8(0.724)$ & $18.9(0.491)$ & $\mathrm{Z}$ \\
\hline S5N* & $64.5(3.22)$ & $40.8(0.647)$ & $15.8(0.233)$ & $\mathrm{Z}$ \\
\hline $\mathrm{S} 5 \mathrm{~N}$ & $36.5(2.31)$ & $34.6(0.856)$ & $14.9(0.439)$ & $\mathrm{BC}$ \\
\hline $\mathrm{S} 7 \mathrm{H}^{*}$ & $629(364)$ & $154(2.196)$ & 76.3 (1.67) & $\mathrm{Z}$ \\
\hline $\mathrm{S} 7 \mathrm{H}$ & $66.2(2.72)$ & $53.3(0.501)$ & $51.3(0.790)$ & $\mathrm{AB}$ \\
\hline
\end{tabular}


1

Comparison of mean exponential phase specific-growth rates between microalgal strains grown under photoautotrophic, mixotrophic, and heterotrophic conditions. Error bars reflect standard error of the mean.

Comparison of mean $(n=3)$ exponential phase specific-growth rates between microalgal strains grown under photoautotrophic, mixotrophic, and heterotrophic conditions. Error bars reflect standard error of the mean.

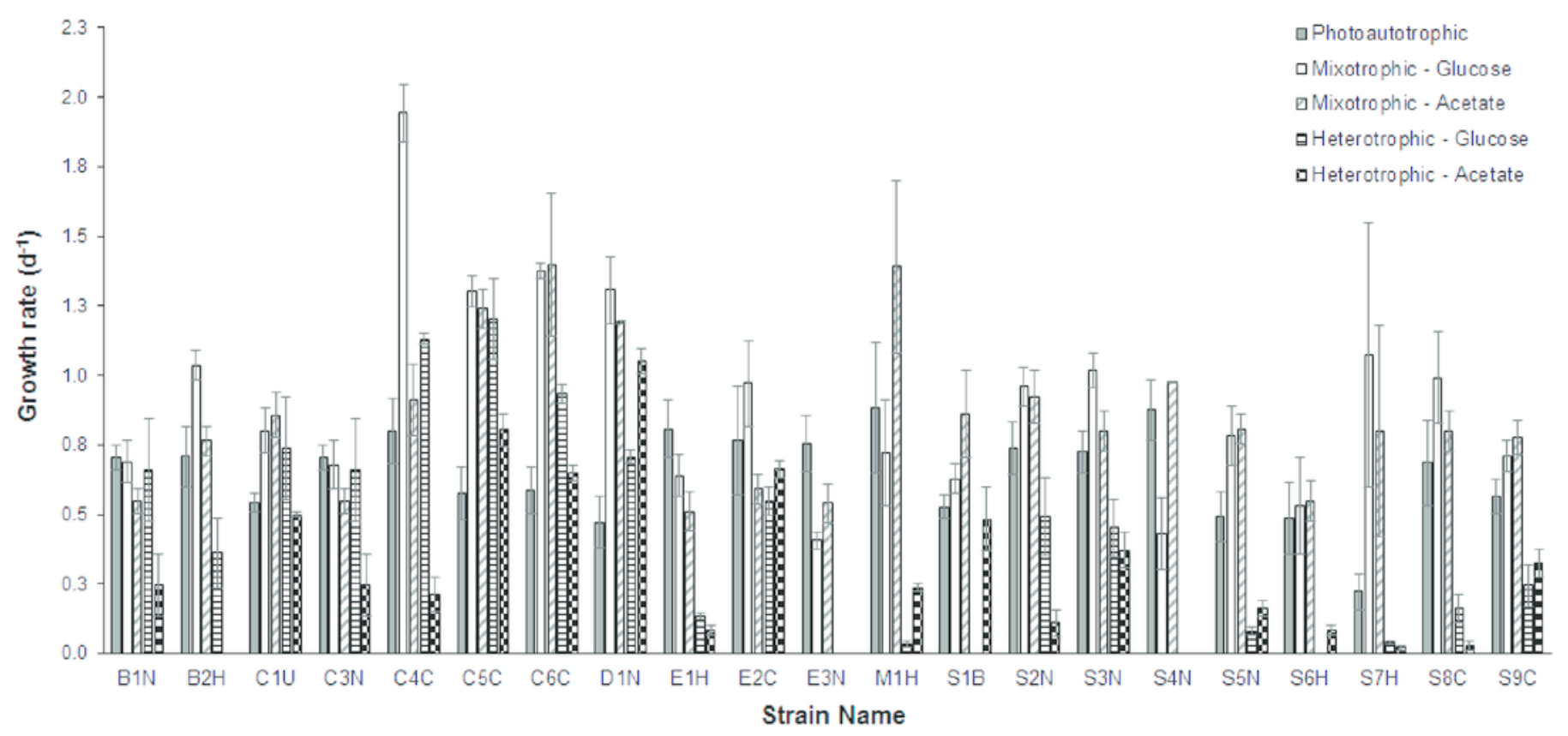


2

Comparison of trophic profiles among strains using cluster analysis based on unweighted pair-group average (UPGMA) using the Bray-Curtis Similarity Index showing Bootstrap analysis values $(\mathrm{N}=1000)$ at each node.

Comparison of trophic profiles (photoautotrophic, mixotrophic, and heterotrophic) among strains using cluster analysis based on unweighted pair-group average (UPGMA) using the Bray-Curtis Similarity Index showing Bootstrap analysis values $(N=1000)$ at each node.

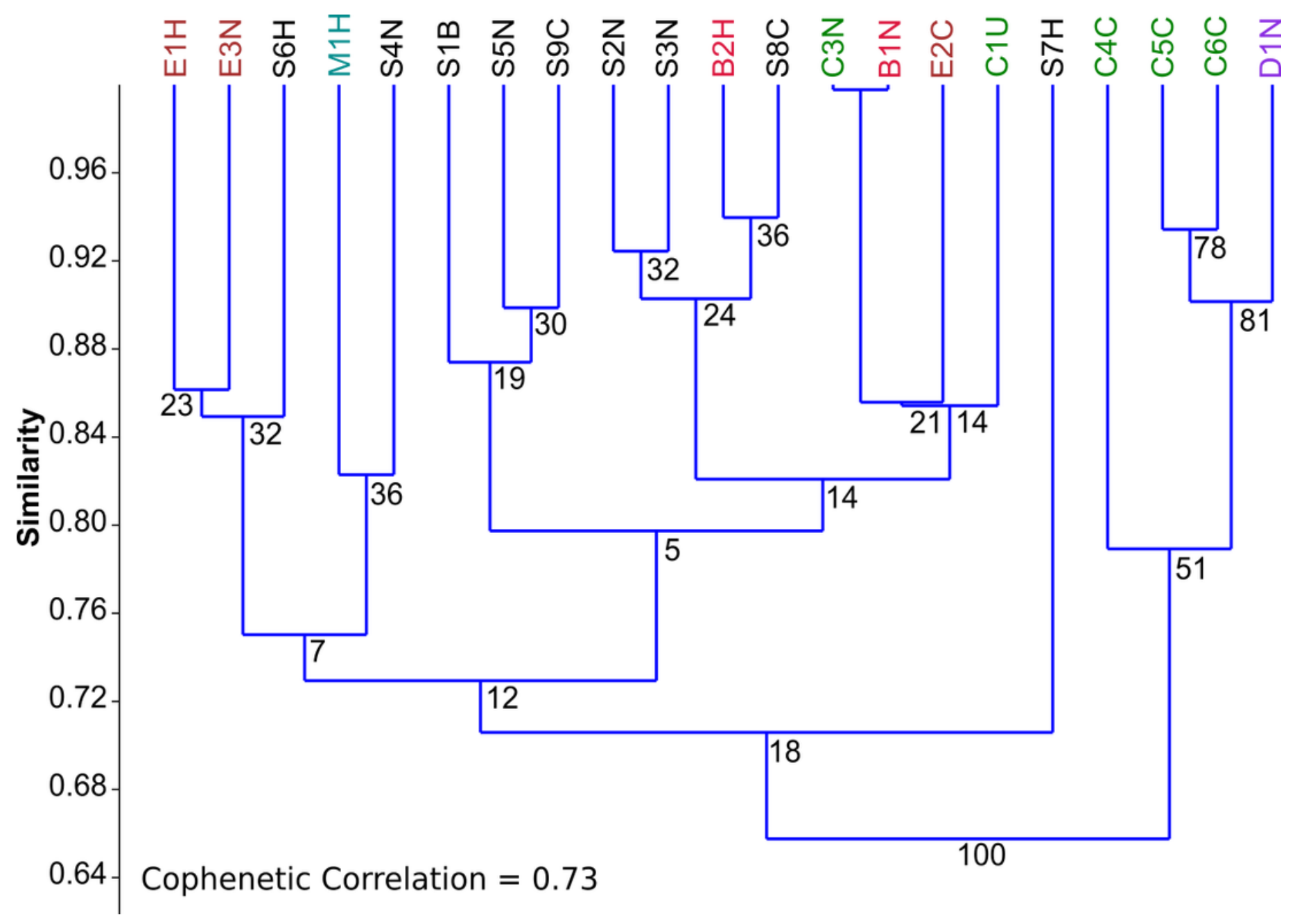


Figure 3 (on next page)

PCA biplots A, B and C.

Principal component analysis (PCA) biplots of fatty acid methyl esters from microalgal strains grown under photoautotrophic (A) mixotrophic (acetate) (B) and mixotrophic (glucose) (C) conditions. Fatty acids are represented by green lables and algal strains are indicated in blue by their strain identification. Strains with an asterisk $(*)$ denote axenic status. 


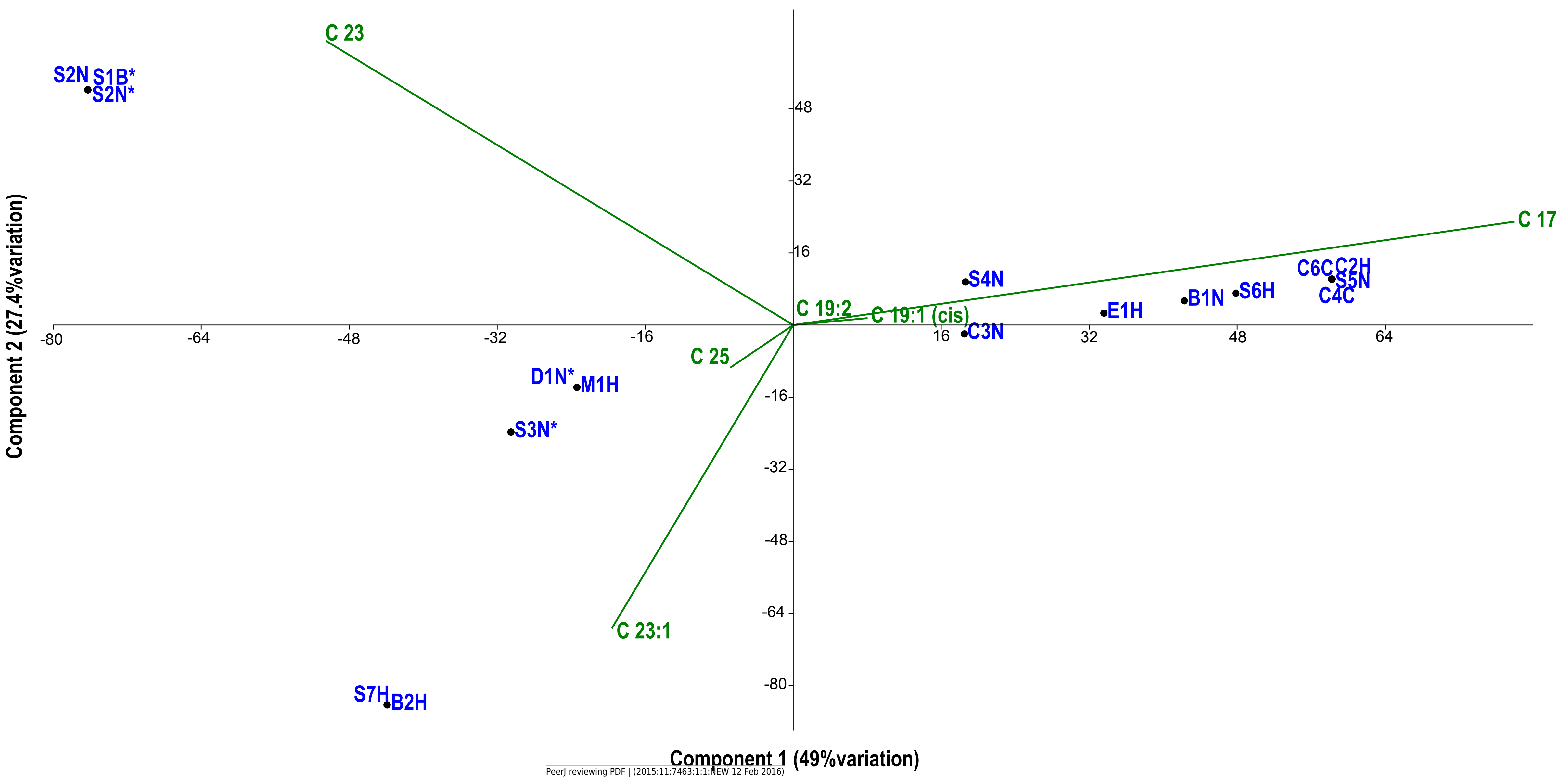

-B1N $\bullet$ S6H C6C5N

S2N ${ }^{-S 1 B} \mathrm{~N}^{*}$ 
Mì

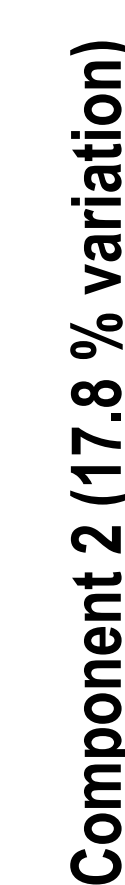

sं̀

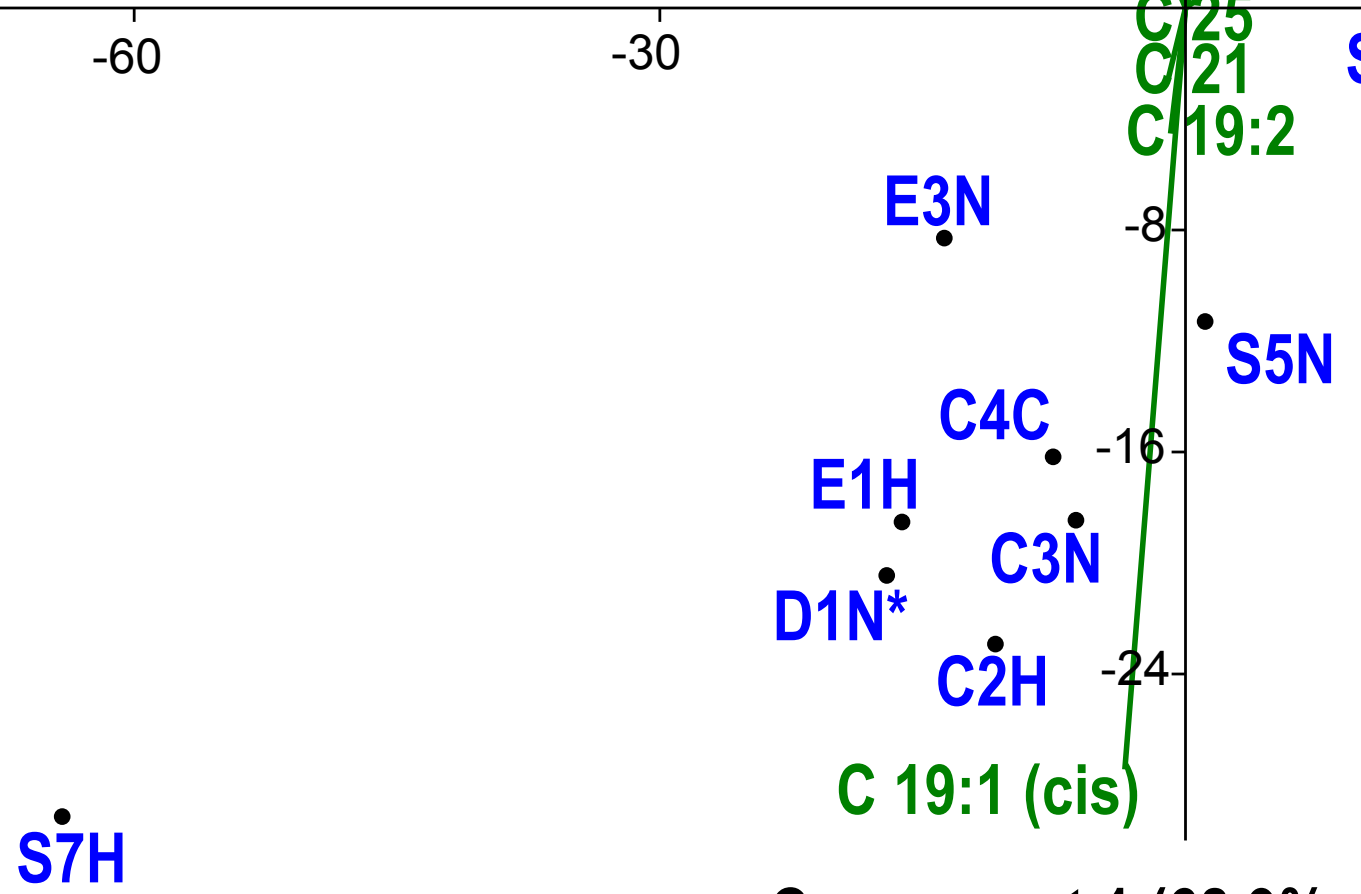


.$S 4 N$

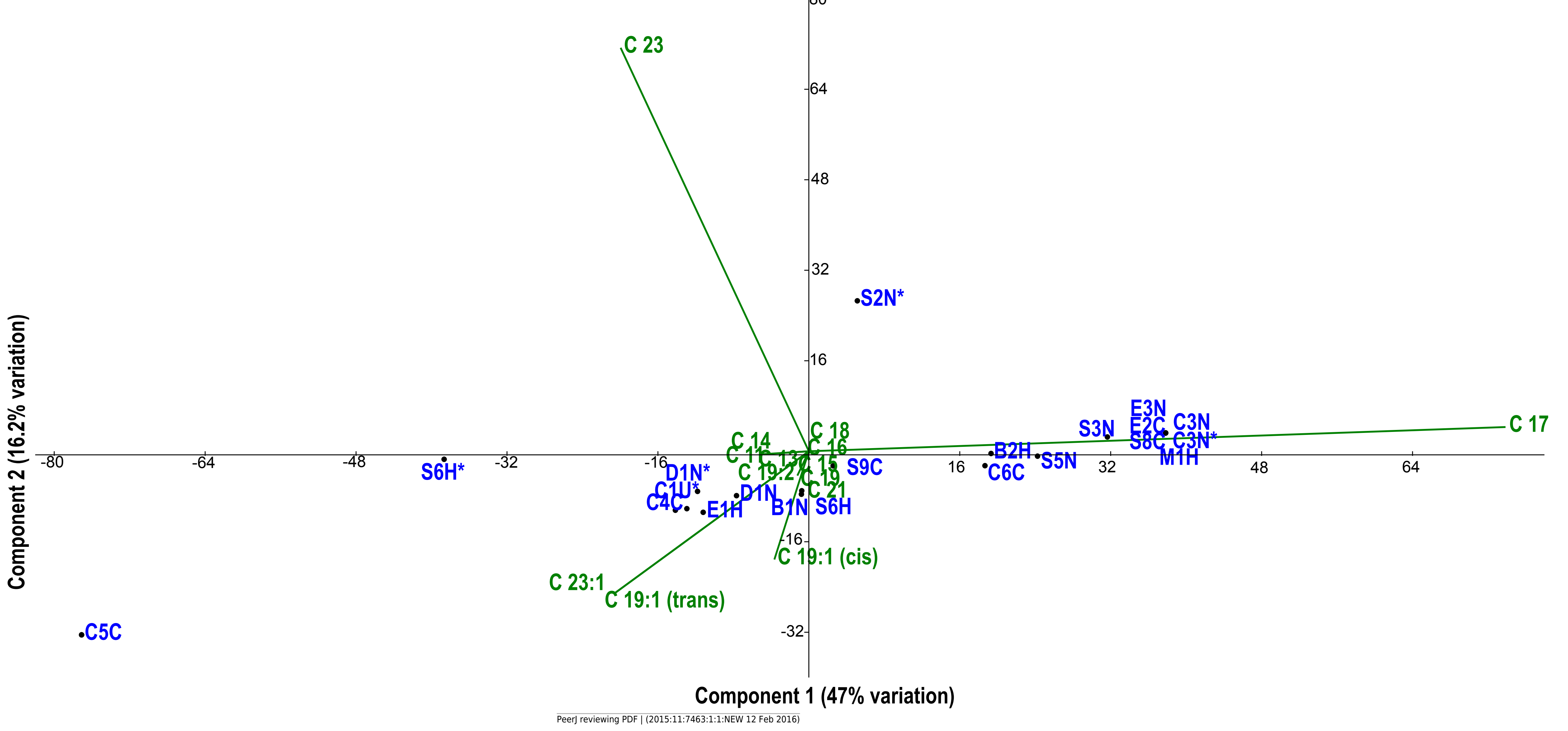

